\title{
A hybrid simulation technique for electrothermal studies of two-dimensional GaN-on- SiC high electron mobility transistors
}

Qing Hao, Hongbo Zhao, and Yue Xiao

Citation: Journal of Applied Physics 121, 204501 (2017); doi: 10.1063/1.4983761

View online: http://dx.doi.org/10.1063/1.4983761

View Table of Contents: http://aip.scitation.org/toc/jap/121/20

Published by the American Institute of Physics

\section{Articles you may be interested in}

Vertical transport in isotype InAIN/GaN dipole induced diodes grown by molecular beam epitaxy Journal of Applied Physics 121, 205702 (2017); 10.1063/1.4983767

Photo-induced changes of the surface band bending in GaN: Influence of growth technique, doping and polarity Journal of Applied Physics 121, 205307 (2017); 10.1063/1.4983846

Infrared dielectric functions, phonon modes, and free-charge carrier properties of high- $\mathrm{Al}_{-}-\mathrm{content} \mathrm{Al}_{\mathrm{X}} \mathrm{Ga}_{1-\mathrm{X}} \mathrm{N}$ alloys determined by mid infrared spectroscopic ellipsometry and optical Hall effect Journal of Applied Physics 121, 205701 (2017); 10.1063/1.4983765

The asymmetric band structure and electrical behavior of the $\mathrm{GdScO}_{3} / \mathrm{GaN}$ system Journal of Applied Physics 121, 205303 (2017); 10.1063/1.4983559

Optical properties of AIGaN nanowires synthesized via ion beam techniques Journal of Applied Physics 121, 205901 (2017); 10.1063/1.4984015

A first-principles study of carbon-related energy levels in GaN. I. Complexes formed by substitutional/interstitial carbons and gallium/nitrogen vacancies

Journal of Applied Physics 121, 195701 (2017); 10.1063/1.4983452

\section{AlP $\mid \begin{aligned} & \text { Journal of } \\ & \text { Applied Physics }\end{aligned}$}

Save your money for your research.

It's now FREE to publish with us no page, color or publication charges apply.
Publish your research in the Joumal of Applied Physics

to claim your place in applied

physics history. 


\title{
A hybrid simulation technique for electrothermal studies of two-dimensional GaN-on-SiC high electron mobility transistors
}

\author{
Qing Hao, ${ }^{\text {a) }}$ Hongbo Zhao, and Yue Xiao \\ Department of Aerospace and Mechanical Engineering, University of Arizona, Tucson, Arizona 85712, USA
}

(Received 13 December 2016; accepted 7 May 2017; published online 22 May 2017)

\begin{abstract}
In this work, a hybrid simulation technique is introduced for the electrothermal study of a twodimensional GaN-on-SiC high electron mobility transistor. Detailed electron and phonon transport is considered by coupled electron and phonon Monte Carlo simulations in the transistor region. For regions away from the transistor, the conventional Fourier's law is used for thermal analysis to minimize the computational load. This hybrid simulation strategy can incorporate the physical phenomena over multiple length scales, including phonon generation by hot electrons in the conduction channel, frequency-dependent phonon transport in the transistor region, and heat transfer across the whole macroscale device. Published by AIP Publishing.
\end{abstract}

[http://dx.doi.org/10.1063/1.4983761]

\section{INTRODUCTION}

In recent years, tremendous efforts have been dedicated to GaN high electron mobility transistors (HEMTs) for highpower and high-frequency applications. In a GaN HEMT, two-dimensional electron gas (2DEG) is formed on the interface between $\mathrm{GaN}$ and its ternary alloy. With its high carrier mobility and carrier density, such 2DEG can transport a large current for power electronics. Currently, GaN HEMTs have demonstrated a record-breaking $40 \mathrm{~W} / \mathrm{mm}$ RF power output level as power amplifiers ${ }^{1}$ and $>10 \mathrm{kV}$ breakdown voltages as power-switching devices. ${ }^{2}$ However, such device performance is largely restricted by significant overheating within the device, which would dramatically reduce the charge carrier mobility and thus lower the output current. ${ }^{3}$ Due to the challenges in thermal management, commercial GaN HEMTs are still limited to $2-4 \mathrm{~W} / \mathrm{mm}$ output power. The strong overheating will also shorten the lifetime of GaN devices, which largely affects their long-term applications. ${ }^{4}$

In principle, the electrothermal simulation of a $\mathrm{GaN}$ HEMT should incorporate various physical phenomena occurring at different length scales. At the submicron scale, heat is first generated as acoustic or optical phonons emitted by hot electrons that gain energy from an ultrahigh electric field along the conduction channel. Nonpropagating optical phonons can decay into acoustic phonons that eventually carry heat across the whole millimeter- or centimeter-sized device. For simulations, the major challenge lies in that detailed electron and phonon transport cannot be considered for the whole macroscale device due to the huge computational load. Simplifications must be taken and may lead to large discrepancy from the experimental data.

In the simplest treatment, the conventional steady-state heat conduction equation is used, i.e., $k \nabla^{2} T+H=0$. Here, $k$ is the thermal conductivity and $T$ is the temperature. The volumetric heat generation rate, $H$, is computed as the Joule

\footnotetext{
a) Author to whom correspondence should be addressed: qinghao@email. arizona.edu.
}

heat $\mathbf{J} \cdot \mathbf{E}$. The used current density $\mathbf{J}$ and the electric field $\mathbf{E}$ are provided by the electron modeling based on the driftdiffusion model that assumes electrons to be in thermal equilibrium with the lattice. ${ }^{5}$ Examples of this treatment can be found in many studies. ${ }^{6-10}$ Multiple problems should be pointed out in this treatment. First, the heat conduction equation is based on the Fourier's law that becomes invalid at the micro- to nano-scale. In this case, the Boltzmann transport equation (BTE) of phonons should be used to replace the analysis using Fourier's law. ${ }^{11}$ Second, the employed driftdiffusion model does not consider the nonequilibrium between hot electrons, optical phonons, and acoustic phonons. Third, Joule heating is inaccurate for heat generation at small scales. The location for the peak heat generation rate may be away from the peak $H$ location because hot charge carriers may further travel a few mean free paths (MFPs) before passing their energy to phonons. ${ }^{12,13}$

In a more accurate treatment, the hydrodynamic model is used to describe the electron transport. Two thermodynamic systems, i.e., electrons and acoustic phonons, are assumed to have their own temperatures to be solved for. ${ }^{14,15}$ The electron modeling is coupled with the phonon energy conservation equation, in which $H$ in the heat conduction equation is replaced by a collision term between electrons and phonons. This treatment neglects the important contribution from optical phonons. ${ }^{16,17}$ In recent studies, longitudinal optical (LO) phonons are added as the third thermodynamic system with their own temperature. ${ }^{18}$ However, the Fourier's law used in the phonon equation is still not valid for a submicron device and may lead to inaccurate results.

To further improve the accuracy, the hydrodynamic model should be replaced by electron Monte Carlo (MC) simulations that track the movement and scattering of individual electrons. The electron density, energy, and drift velocity, as extracted from electron MC simulations, are input into the energy conservation equations for phonons. ${ }^{19,20}$ Optical phonons have also been added in such calculations. In a rough treatment, the energy exchange between electrons and phonons can also be counted and directly used as $H$ in the heat 
conduction equation. ${ }^{21,22}$ Such electron MC simulations have been continuously improved by considering the hot-phonon effect. $^{23,24}$ The required local temperature can be obtained from coupled thermal simulations ${ }^{25}$ or experiments. ${ }^{26-28}$ Despite better predictions of electron transport with electron MC simulations, the usage of the Fourier's law for phonons can still lead to large errors.

To provide the most accurate temperature predictions, this work introduces coupled electron and phonon MC simulations for a GaN HEMT. The electron MC simulations will track the movement and scattering of individual charge carriers and provide phonons emitted by hot electrons. The net phonon emission will be further input into the phonon MC simulations to update the phonon temperatures that further affect electron scattering in electron MC simulations. The two simulations are thus carried out in an iterative way to achieve self-consistence. Energy-dependent electron and phonon transport can be fully considered in these MC simulations. To further predict the temperature profile of the entire macroscale device, the phonon MC simulation is coupled with the conventional Fourier's law analysis for regions away from the transistor. Figure 1 summarizes the scheme of the proposed "hybrid" simulation technique that can fully incorporate energy-dependent phonon and electron transport. The technique can also be easily extended to complicated device structures for thermal studies.

\section{SIMULATION SCHEME}

The transport of charge carrier and heat in device is governed by the corresponding BTE, which considers a timedependent distribution function in the position-velocity phase space. Under the relaxation time approximation, the electron BTE is

$$
\frac{\partial f}{\partial t}+\mathbf{v} \cdot \nabla_{\mathbf{r}} f+\mathbf{F} \cdot \nabla_{\mathbf{v}} f=\left[\frac{\partial f}{\partial t}\right]_{\text {coll }},
$$

where the right hand side is the collision term. As the heat carrier in the wave packet form, the phonon BTE is

$$
\frac{\partial f}{\partial t}+\nabla_{\mathbf{k}} \omega(\mathbf{k}, p) \cdot \nabla_{\mathbf{r}} f=\left[\frac{\partial f}{\partial t}\right]_{\text {coll }},
$$

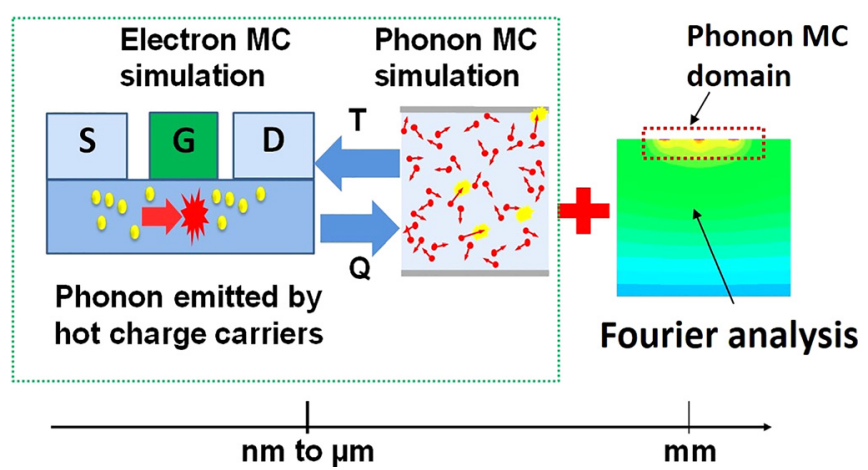

FIG. 1. Scheme of multi-length scale electrothermal simulations of a GaN HEMT. where the phonon angular frequency $\omega$ is a function of phonon wave vector $\mathbf{k}$, and $p$ indicates the phonon branch. In these equations, the distribution function $f$ is a function of the location, traveling direction, energy, and branch of individual carriers. Considering the multidimensional nature of $f$, directly solving the BTE to obtain $f$ can be extremely challenging. In this case, the MC simulations are more favorable as an alternative method to solve the BTE.

In both electron and phonon MC simulations, bundles of electrons or phonons are initially randomly distributed across the computation domain. The computational domain is divided into many spatial bins called subcells. The initial conditions for each bundle (e.g., energy, velocity, subband, and valley for electrons) are set according to the equilibrium distribution of electrons or phonons. Within each time step, the electrons or phonons move classically. At the end of each time step, scattering is determined randomly according to the scattering rates of different mechanisms. To capture the scattering processes of a carrier, the time step used in MC simulations should be much shorter than the typical relaxation time for the corresponding carriers. The local electron and phonon temperatures are computed by the energy density of these carriers within each subcell. For steady-state studies, these simulations converge when the temperature profiles of electrons, optical phonons, and acoustic phonons are no longer changed during iterations. By tracking a large number of electron or phonons, MC simulations can approach BTE solutions statistically. ${ }^{29}$

\section{A. Electron MC simulations}

Electron MC simulations are used to predict the heat generation due to phonons emitted by the hot 2DEG at the interface between $\mathrm{GaN}$ and AlGaN alloys. Three conduction band valleys are included in electron MC simulations. From low to high energy, they are the $\Gamma_{1}$ valley, the $U$ valley (the valley between $L$ and $M$ points), and the $\Gamma_{3}$ valley. All valleys are represented with an analytical nonparabolic band following the Kane's model, ${ }^{30}$ i.e., $E_{i}\left(1+\alpha_{i} E_{i}\right)=\hbar^{2} k^{2} / 2 m_{0, i}^{*}$, where $E$ is the kinetic energy of electrons, $m_{0}^{*}$ is the electron effective mass at the band edge, and $i$ is the index for the three valleys. In this model, the effective mass is kinetic-energy-dependent as $m^{*}=m_{0}^{*}(1+2 \alpha E)$. Using the energy-dependent effective mass allows the electron to be treated as in a parabolic band. ${ }^{31}$ Due to quantum confinement, discrete subbands are generated for each valley. ${ }^{32}$ By solving the Schrödinger-Poisson equation for the $\mathrm{AlGaN} / \mathrm{GaN}$ heterostructure, the self-consistent subband energy structure can be calculated. ${ }^{33,34}$ It has been shown that just considering two subbands of the lowest valley can be sufficient for an electric field as high as $4 \times 10^{7} \mathrm{~V} / \mathrm{m} .{ }^{35}$ Three subbands of the lowest valley have been considered, with the second and third subbands separated from the first one by $0.11 \mathrm{eV}$ and $0.15 \mathrm{eV}$, respectively. ${ }^{34}$ These three subbands and their energies are adopted in the electron MC simulations. For the higher two valleys, subbands are not considered because they are not as important for the electric field considered in this work. This simplification for the $\mathrm{U}$ and $\Gamma_{3}$ valleys is also a common practice in electron $\mathrm{MC}$ simulations of $\mathrm{GaN}$ for an electric field of a few $10 \mathrm{MV} / \mathrm{m}$. 
For the 2DEG, the electron scattering mechanisms include polar optical phonon scattering, acoustic deformation potential scattering, and intervalley optical phonon scattering. (See Appendices A 1-A 3 for detailed scattering rate expression.) Under a high electric field, only optical phonon emission is considered and acoustic phonon scattering of electrons is assumed to be elastic. ${ }^{36}$ Similar treatment can be found in previous MC studies on 2DEG of GaN. ${ }^{37}$ Only the topmost longitudinal optical phonons at $91.2 \mathrm{meV}$ are considered as the major scattering mechanism for hot electrons. ${ }^{35,38} \mathrm{It}$ should be noted that 2DEG has slightly decreased electronphonon scattering. ${ }^{35}$ For 2 DEG, it is possible to compute the exact scattering rates as a function of wave vectors by Fermi's golden rule. ${ }^{33,39,40}$ However, this may largely increase the computational load and is not suitable for the hybrid simulation technique. In the simplified treatment, the $3 \mathrm{D}$ electronphonon scattering formulation has been generally adopted in the literature ${ }^{14,19,24,34,35}$ and is justified by good fittings with experiments. Since this work focuses more on coupling phonon MC simulations with electron MC simulations, the electron MC simulations performed are kept the same as existing studies. Table I lists the parameters used in our electron MC simulations, which follows the work by Li et al. ${ }^{35}$

The electric field applied to the 2DEG can be calculated self-consistently by coupling the electron MC simulation with the Poisson equation solver. The polarization charges at the surface of $\mathrm{AlGaN}$ and at the $\mathrm{AlGaN} / \mathrm{GaN}$ interface, the electron charge density of 2DEG, and the applied transistor terminal potentials are among the factors that need to be considered for the Poisson solver. Details of the treatment are given in Appendix B.

\section{B. Phonon MC simulations}

Similar to electron MC simulations, the movement and scattering events of all phonons are tracked in phonon $\mathrm{MC}$ simulations. ${ }^{29,41,42}$ Conventionally, phonon MC simulations are not considered for microscale devices due to the high computational load. This issue has been resolved with a new deviational phonon MC technique developed by Péraud and Hadjiconstantinou. ${ }^{43,44}$ This new technique only tracks phonons related to the deviation of the phonon distribution function $f$ from the equilibrium $f_{0}$ (i.e., Bose-Einstein distribution) at a reference temperature. This allows phonon MC simulations for electronic devices with sizes up to $\sim 10 \mu \mathrm{m}$.

Three input parameters are required by phonon MC simulations: (1) the phonon dispersion for each material,

TABLE I. Parameters used in electron MC simulations.

\begin{tabular}{lcc}
\hline \hline Parameter (Unit) & Symbol & Value \\
\hline Electron density in 2DEG $\left(\mathrm{cm}^{-2}\right)$ & $n_{2 D}$ & $1.0 \times 10^{13}$ \\
Electron effective mass $\left(m_{0}\right)$ & $m_{i}{ }^{*}$ & $0.21,0.25,0.40$ \\
Valley minimum energy $(\mathrm{eV})$ & $E_{c, i}$ & $0,1.95,2.1$ \\
Nonparabolicity $\left(\mathrm{eV}^{-1}\right)$ & $\alpha_{i}$ & $0.19,0.1,0$ \\
Dielectric constant $\left(\varepsilon_{0}\right)$ & $\varepsilon_{s}, \varepsilon_{\infty}$ & $9.5,5.35$ \\
Mass density $\left(\mathrm{g} / \mathrm{cm}^{3}\right)$ & $\rho$ & 6.095 \\
Acoustic deformation potential $(\mathrm{eV})$ & $D_{a}$ & 8.0 \\
Intervalley deformation potential $(\mathrm{eV} / \mathrm{cm})$ & $D_{i j}$ & $1.0 \times 10^{9}$ \\
\hline \hline
\end{tabular}

(2) phonon scattering rates, and (3) interfacial phonon transmissivity. The exact treatment of internal and interfacial phonon scattering can be found in the literature. ${ }^{29,41,42}$ To simplify, an isotropic sine-shaped phonon dispersion (Bornvon Karman dispersion) is used for all materials. The sine dispersion is better than the Debye model, as it captures the dispersion features at both low and high ends of the frequency range. The dispersion is characterized by two parameters, the maximum phonon angular frequency $\omega_{\max }$ and maximum wave vector $k_{0}$ as $\omega(k)=\omega_{\max } \sin \left(\pi k / 2 k_{0}\right)$. Here, $k_{0}$ and the equivalent atomic distance $a_{\mathrm{D}}$ can be calculated using $k_{0}=\pi / a_{D}=\left(6 \pi^{2} N\right)^{1 / 3}$, with $N$ as the volumetric density of primitive cells. The essential phonon scattering mechanisms in all related materials are impurity scattering and the Umklapp process of the phonon-phonon scattering. The rates are expressed as $W_{\text {imp }}(\omega)=A \omega^{4}$ and $W_{U}(\omega)=B_{1} \omega^{2} T$ $\exp \left(-B_{2} / T\right)$, respectively. ${ }^{45,46}$ Parameters of all materials, obtained by fitting measured thermal conductivities, are listed in Table II. In Fig. 2, we plotted the thermal conductivity accumulation function calculated from our fitting for the four materials within HEMTs. Accumulation function at length $x$ measures the percentage of the lattice thermal conductivity contributed by all phonons with MFP less than $x$. This gives a clear overview of the MFP spectrum of phonons for their contribution to heat conduction. Experimental data for $\mathrm{GaN}, 4 \mathrm{H}-\mathrm{SiC}$, and $\mathrm{AlN}$ at two different temperatures ${ }^{47}$ are also shown in Figs. 2(a)-2(c). Despite the difference between $4 \mathrm{H}-\mathrm{SiC}$ and $6 \mathrm{H}-\mathrm{SiC}$, our model agrees with the experimental results in general. Figure 2(a) shows that about $70 \%$ of the lattice thermal conductivity of $\mathrm{GaN}$ is contributed by phonons with MFP less than $2 \mu \mathrm{m}$. In physics, the percentage of phonons not scattered for a traveling distance $L$ is given as $\exp (-L / \Lambda)$ for a phonon MFP of $\Lambda$. Based on this, majority phonons emitted by hot electrons have been scattered when the distance from the hot spot is a few micrometers. In this case, the Fourier's law can be applied when it is several micrometers away from the transistor. This helps the determination of the size of the domain of the phonon MC simulation.

Within each time step of phonon MC simulations, new acoustic phonons are emitted from the hot spot regions. These acoustic phonons mainly originate from the decay of optical phonons emitted by hot electrons. In steady states, the total energy of acoustic phonons matches the energy of emitted optical phonons within each subcell. The energy and branch of emitted acoustic phonons are randomly set based on the local temperature of optical phonons, following similar phonon setting based on a given temperature. ${ }^{29,41,42}$

TABLE II. Fitted phonon dispersion and scattering parameters for materials in the HEMT device.

\begin{tabular}{lcccc}
\hline \hline Parameter (Unit) & $\mathrm{GaN}$ & $\mathrm{Al}_{0.3} \mathrm{Ga}_{0.7} \mathrm{~N}$ & $\mathrm{AlN}$ & $\mathrm{SiC}$ \\
\hline$k_{0}\left(10^{9} \mathrm{~m}^{-1}\right)$ & 10.94 & 10.99 & 11.19 & 8.94 \\
$\omega_{\max }\left(10^{13} \mathrm{rad} / \mathrm{s}\right)$ & 3.50 & 3.99 & 5.18 & 7.12 \\
$a_{D}(\AA)$ & 2.87 & 2.86 & 2.81 & 3.51 \\
$\mathrm{~A}\left(10^{-45} \mathrm{~s}^{3}\right)$ & 5.26 & 817 & 10.5 & 1.00 \\
$B_{l}\left(10^{-19} \mathrm{~s} / \mathrm{K}\right)$ & 1.10 & 0.421 & 0.728 & 0.596 \\
$B_{2}(\mathrm{~K})$ & 200.0 & 239.1 & 287.5 & 235.0 \\
\hline \hline
\end{tabular}



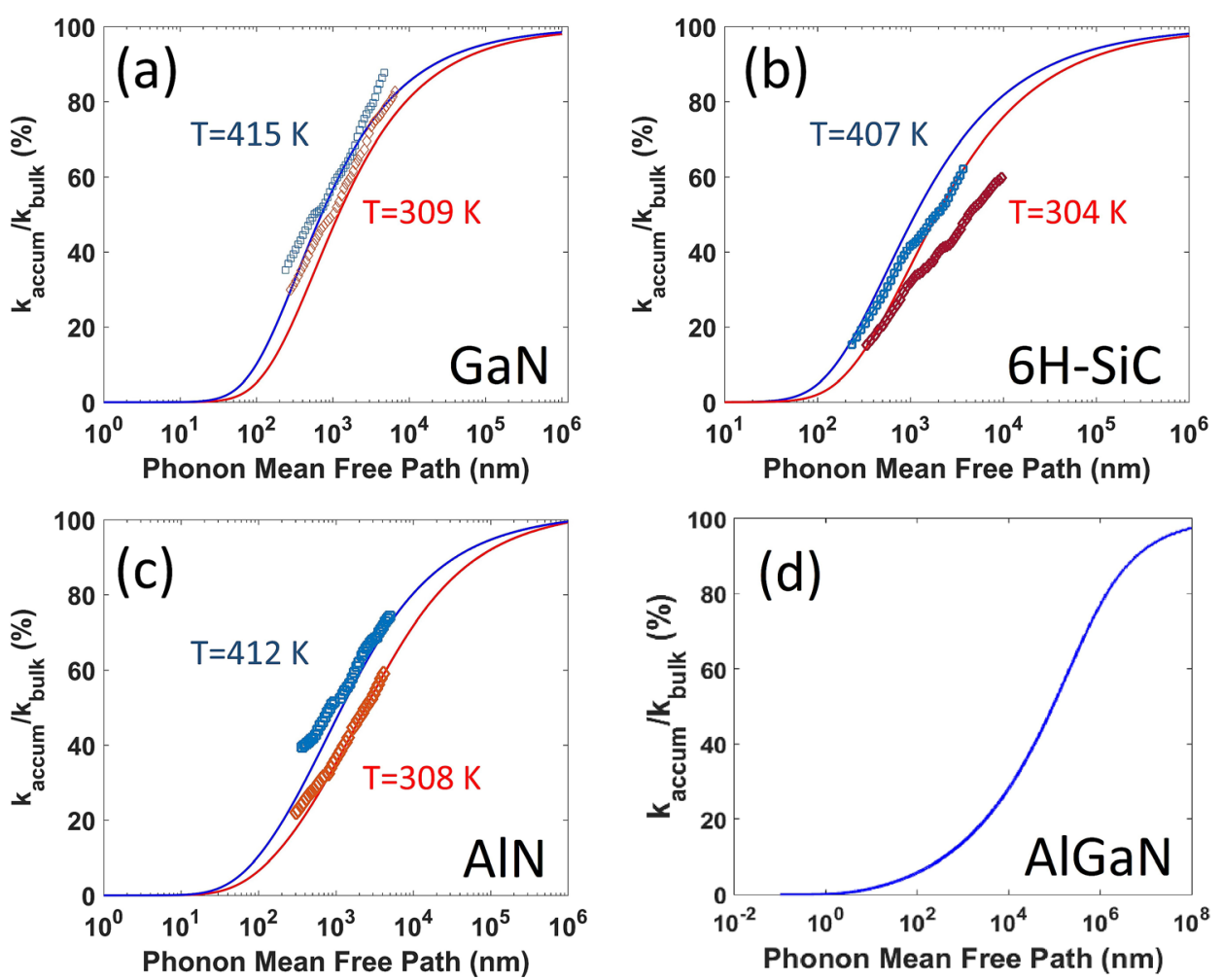

FIG. 2. Thermal conductivity accumulation function for device materials. (a) GaN, (b) $6 \mathrm{H}-\mathrm{SiC}$, (c) $\mathrm{AlN}$, and (d) $\mathrm{Al}_{0.3} \mathrm{Ga}_{0.7} \mathrm{~N}$. The squares represent experimental data from Freedman et al. $^{47}$ The lines are calculation from our fitting parameters at the experimental temperature for (a)-(c) and at $300 \mathrm{~K}$ for $(\mathrm{d})$.
In phonon $\mathrm{MC}$ simulations, phonons are assumed to be in thermal equilibrium with the local temperature on the interface between the phonon MC domain and Fourier domain. The detailed treatment of such an interface can be found elsewhere. ${ }^{43}$ The temperature profile on this domain interface is determined by iterating the phonon MC simulation and Fourier's law analysis, which will be discussed in Section II D. For the rest of the phonon MC domain boundary (i.e., the top surface of the device), diffusive reflection is enforced for incident phonons.

\section{Optical phonon temperature determination}

One important issue of the electrothermal simulation is how to effectively determine the optical phonon temperature. Considering the electron, acoustic phonon, and optical phonon subsystems in the electron MC simulation, the optical phonon energy conservation leads to $19,48,49$

$$
C_{O} \frac{\partial T_{O}}{\partial t}=\frac{3 n K_{B}}{2} \frac{T_{e}-T_{O}}{\tau_{e-O}}+\frac{n m v_{d}^{2}}{2 \tau_{e-O}}-C_{O} \frac{T_{O}-T_{A}}{\tau_{O-A}}
$$

where $C_{O}$ is the specific heat of optical phonons, $n$ is the electron density, $v_{d}$ is the electron drift velocity, and $T_{e}, T_{A}$, and $T_{O}$ are the position-dependent temperatures for electrons, acoustic phonons, and optical phonons, respectively. For 2DEG, both $C_{O}$ and $n$ are evaluated for a unit area on the GaN/AlGaN interface. $\tau_{e-O}$ and $\tau_{O-A}$ are the averaged relaxation time for electron-optical phonon scattering and opticalacoustic phonon scattering, respectively. $\tau_{e-O}$ depends on the local temperature of subcells and $\tau_{O-A}$ is chosen to be 2 ps. In the steady state, the left side of Eq. (3) is zero. In this case, Eq. (3) is reduced to

$$
\frac{3 n K_{B}}{2} \frac{T_{e}-T_{O}}{\tau_{e-O}}+\frac{n m v_{d}^{2}}{2 \tau_{e-O}}=C_{O} \frac{T_{O}-T_{A}}{\tau_{O-A}}
$$

The temperature of electrons and acoustic phonons can be obtained from the electron and phonon MC simulations, respectively. The drift velocity $v_{d}$ can also be provided by the electron MC simulation. The temperature of the optical phonons can then be derived using Eq. (4) and further used in electron $\mathrm{MC}$ simulations to determine the scattering rate of polar optical phonons.

\section{Coupling phonon MC simulations with Fourier's law calculation}

Despite the enlarged phonon MC simulation domain with the new deviational MC technique, the domain size is still much smaller than the sub-millimeter device size. For regions away from the transistor region, Fourier's law analysis based on ANSYS simulations is thus used and coupled with phonon MC simulations. The overall device simulation involves coupled electron MC simulation, phonon MC simulation, and Fourier's law analysis. The electron MC simulation predicts optical phonon generation but needs both acoustic and optical phonon temperatures at the channel to determine the electron scattering rates. The phonon $\mathrm{MC}$ simulation outputs acoustic phonon temperatures based on phonon generation by $2 \mathrm{DEG}$ and phonon temperature on the interface between the Fourier and phonon MC domains. Fourier's law analysis employs heat generation from electron MC simulations and outputs the temperature profile across the whole device, with the transistor region further refined by phonon MC simulations. Starting with a guessed acoustic phonon temperature profile along the 2DEG channel, electron MC simulation produces heat generation rate per unit area on the GaN/AlGaN interface. The 
Fourier's law analysis in turn generates $T_{A}$ at the phonon MC domain boundary. Phonon MC simulation can then be used to refine the $T_{A}$ profile close to the transistor. Full convergence is achieved when the $T_{A}$ profile at the interface between the phonon MC simulation domain and Fourier domain changes less than $5 \%$ of the maximum temperature rise between iterations in the transistor. Normally, within 10 iterations the convergence can be reached. The vertical range of the phonon MC simulation domain normally includes all the thin layers of HEMT and the top few micrometers of the substrate, and the horizontal range includes the transistor and few micrometers away from the transistors from both ends. As can be seen in Section III, $T_{A}$ close to the phonon MC domain boundaries already agrees with the analysis based on Fourier's law. The size of the phonon MC domain is also in line with other phonon $\mathrm{MC}$ simulations. ${ }^{50}$

\section{RESULTS AND DISCUSSION}

A 2D GaN HEMT is simulated in this work. The fourlayered structure of the device is illustrated in Fig. 3. From the top to the bottom, the layers are a 30-nm-thick AlGaN layer, a $2.5-\mu \mathrm{m}$-thick GaN layer, a 60 -nm-thick AlN nucleation layer, and the substrate $\mathrm{SiC}$ with $500 \mu \mathrm{m}$ thickness. The source, gate, and drain are on top of the AlGaN layer and are all $1 \mu \mathrm{m}$ wide and separated by $1 \mu \mathrm{m}$. The horizontal length of the whole device is chosen to be $500 \mu \mathrm{m}$.

The electron MC simulation is only carried out for the 2DEG between the source and drain, with a channel length of $3 \mu \mathrm{m}$. Both the source and the gate are grounded, and a $15 \mathrm{~V}$ voltage is applied to the drain. The electric field is peaked right at the edge of the gate on the drain side.

Figure 4 compares the classic Joule heating with the heat generation rate from electron MC simulations by counting the total energy of emitted phonons per unit time per unit area of 2DEG. The Joule heating is calculated as $\mathbf{J} \cdot \mathbf{E}$ and is the macroscopic version of the energy dissipation of electrons driven by an electric field. Here, the current density $\mathbf{J}$ is obtained at the end of the electron MC simulation. For a small electric field on the left half of Fig. 4, Joule heating does not differ largely from the heat generation rate from phonon emission. However, the peak heat generation rate is overestimated with $\mathbf{J} \cdot \mathbf{E}$. This trend agrees with the early work on Si by Pop et al. ${ }^{12}$

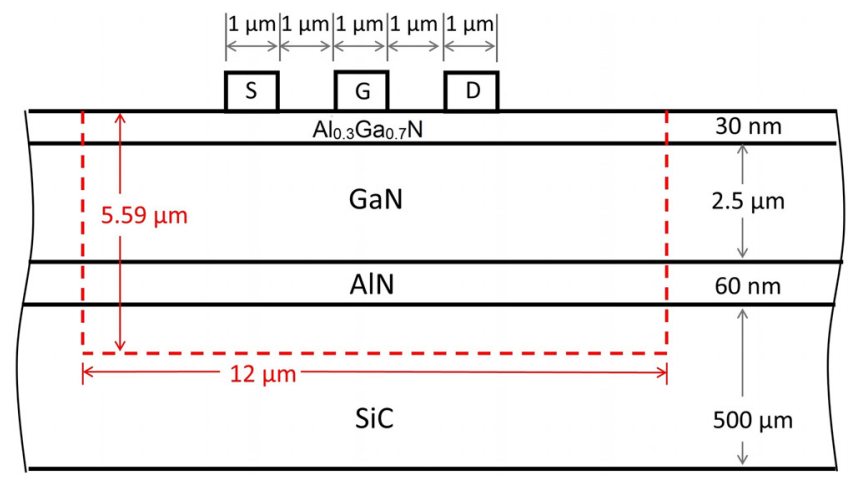

FIG. 3. Schematic diagram of the cross section of the simulated GaN HEMT. The region enclosed by the dashed line is the domain for phonon MC simulations.

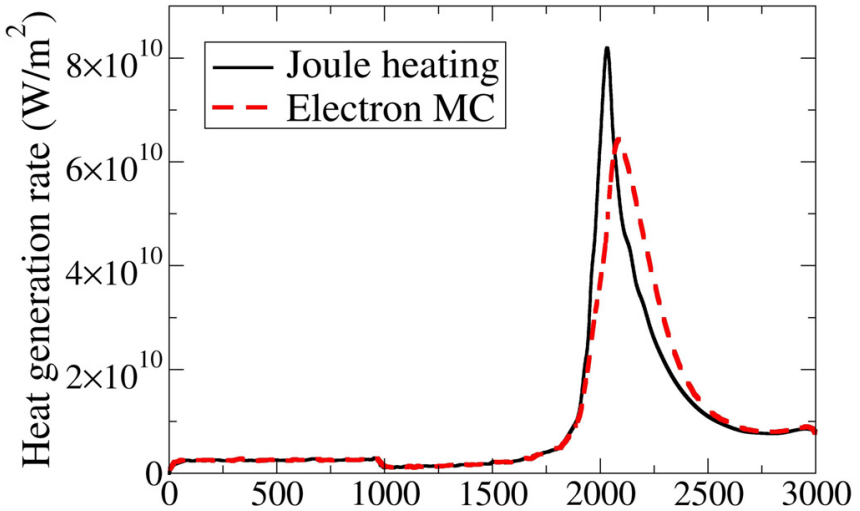

Distance from Drain-side Source edge $(\mathrm{nm})$

FIG. 4. Comparison of the heat generation rate per unit area of 2DEG between Joule heating (black line) and that due to phonon emission calculated from electron MC simulation (red dashed line). The profile is for the region between gate-side edges of the source and drain.

The domain size for phonon $\mathrm{MC}$ simulations is chosen as $12 \mu \mathrm{m}$ long and $5.59 \mu \mathrm{m}$ deep. Horizontally, the center of the gate aligns with the center of the phonon MC simulation domain. The GaN, AlGaN, and AlN layers, as well as the top $3 \mu \mathrm{m}$ thickness of the $\mathrm{SiC}$ substrate, are included in the domain, as shown using a dashed line in Fig. 3. Compared with majority phonon MFPs of less than $2 \mu \mathrm{m}$, the distance between the hot spot and phonon MC domain boundary is sufficiently long so that the Fourier's law becomes valid outside the phonon MC domain. Due to their weak contribution, the thin metal layers of the terminals (i.e., source, drain, and gate) are neglected in the simulation.

Figure 5(a) shows the acoustic phonon temperature profile in the phonon MC simulation domain after convergence. The hot spot is at the gate edge on the drain side, and the
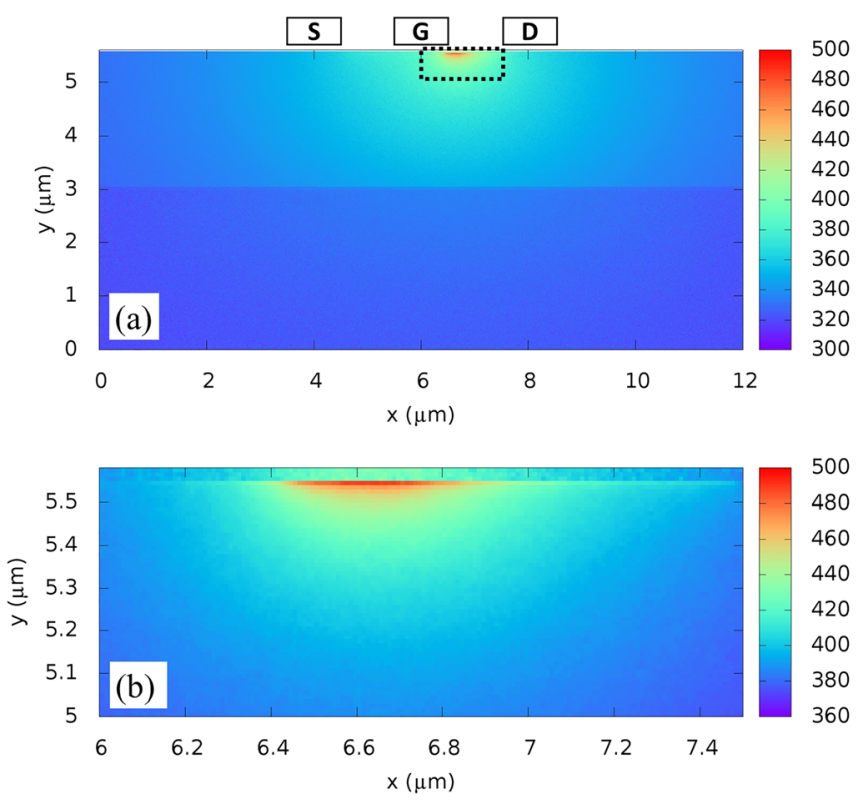

FIG. 5. Acoustic phonon temperature profile from phonon MC simulations. (a) The whole phonon MC simulation domain. (b) Close-up of the hot spot region as enclosed with a dotted line in (a). The origin of both plots is the lower left corner of the phonon MC simulation domain as shown with a dashed line in Fig. 3. 


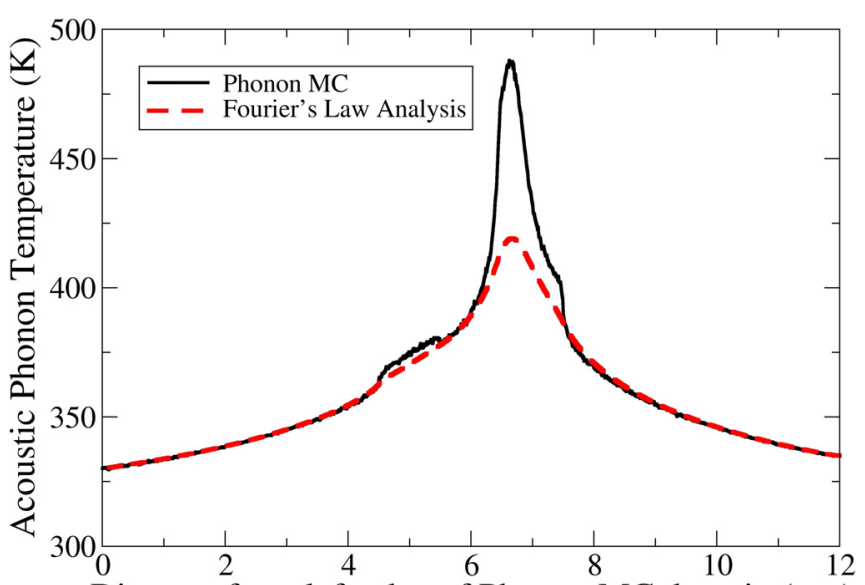

Distance from left edge of Phonon MC domain $(\mu \mathrm{m})$

FIG. 6. Comparison of acoustic phonon temperature at the GaN/AlGaN interface between phonon MC simulation (red line) and Fourier's law calculation (black dashed line). The $\mathrm{x}$ coordinate starts from the left edge of the phonon MC simulation domain as shown with a dashed line in Fig. 3.

highest temperature is around $490 \mathrm{~K}$. The dotted lines in Fig. 5(a) mark the high temperature region of the simulated 2D HEMT, and the time-averaged temperature profile of this region is shown in Fig. 5(b). Figure 6 compares the acoustic phonon temperature profile along the $2 \mathrm{DEG}$ channel, which is evaluated for the GaN subcells on the AlGaN/GaN interface. It is clearly shown that in the main heat generation region where there is heat generation $(4.5<\mathrm{x}<7.5 \mu \mathrm{m})$, the analysis according to Fourier's law can largely underestimate the hot spot temperatures.

\section{CONCLUSION}

We have demonstrated a multi-length scale simulation for 2D GaN HEMTs, which involves coupled electron and phonon MC simulations, and Fourier's law analysis. The heat generation inside the device occurs due to optical phonon emission by hot $2 \mathrm{DEG}$ at the $\mathrm{AlGaN} / \mathrm{GaN}$ interface and is simulated with the electron $\mathrm{MC}$ technique. The hot spot is revealed by the phonon $\mathrm{MC}$ simulation and resides under the gate edge on the drain side, where the electric field reaches its peak value. Away from the transistor, the temperature can be calculated using the Fourier's law. This allows simulations of GaN HEMTs that can be further cooled within the substrate, e.g., active cooling by single-phase or two-phase flows. Unrestricted to $\mathrm{GaN}$ devices, the proposed simulation strategy can be applied to other devices where overheating is critical to the device performance.

\section{ACKNOWLEDGMENTS}

The authors thank Dr. Jean-Philippe M. Péraud for the help with deviational phonon MC simulations and Professor David Broido for the input on the phonon relaxation times within GaN. Hao also thanks Dr. Satish Kumar and Mr. Junda Yan for helpful discussions on GaN HEMTs. An allocation of computer time from the UA Research Computing High Performance Computing (HPC) and High Throughput Computing (HTC) at the University of Arizona is gratefully acknowledged. This material is based on research sponsored by the Defense Advanced Research Agency (DARPA) under agreement number FA8650-15-1-7523. The U.S. Government is authorized to reproduce and distribute reprints for Governmental purposes notwithstanding any copyright notation thereon. The views and conclusions contained herein are those of the authors and should not be interpreted as necessarily representing the official policies or endorsements, either expressed or implied, of the Air Force Research Laboratory (AFRL) and the DARPA or the U.S. Government.

\section{APPENDIX A: ELECTRON SCATTERING RATES}

\section{Polar optical phonon scattering}

Since $\mathrm{GaN}$ is a highly polar material, the scattering between electrons and optical phonons is dominant at temperatures higher than $300 \mathrm{~K} .{ }^{37}$ The scattering rate is treated assuming the Fröhlich interaction. ${ }^{51}$ It has been shown that the $3 \mathrm{D}$ material treatment of polar optical phonon scattering is justifiable for $2 \mathrm{DEG}{ }^{52,53}$ The scattering rates for polar optical phonon emission and absorption are, respectively,

$$
\begin{aligned}
\Gamma_{\mathrm{pop}}^{\mathrm{absorb}}(E)= & \frac{e^{2} \omega_{\mathrm{op}} \sqrt{2 m_{n}{ }^{*}}}{\hbar 8 \pi}\left(\frac{1}{\varepsilon_{\infty}}-\frac{1}{\varepsilon_{0}}\right) \frac{N_{q}}{\sqrt{\gamma(E)}} \\
& \times \ln \frac{\sqrt{\gamma\left(E+\hbar \omega_{\mathrm{op}}\right)}+\sqrt{\gamma(E)}}{\sqrt{\gamma\left(E+\hbar \omega_{\mathrm{op}}\right)}-\sqrt{\gamma(E)}} \\
& \times\left[1+2 \alpha\left(E+\hbar \omega_{\mathrm{op}}\right)\right]
\end{aligned}
$$

and

$$
\begin{aligned}
\Gamma_{\mathrm{pop}}^{\mathrm{emit}}(E)= & \frac{e^{2} \omega_{\mathrm{op}} \sqrt{2 m_{n}^{*}}}{\hbar 8 \pi}\left(\frac{1}{\varepsilon_{\infty}}-\frac{1}{\varepsilon_{0}}\right) \frac{N_{q}+1}{\sqrt{\gamma(E)}} \\
& \times \ln \frac{\sqrt{\gamma(E)}+\sqrt{\gamma\left(E-\hbar \omega_{\mathrm{op}}\right)}}{\sqrt{\gamma(E)}-\sqrt{\gamma\left(E-\hbar \omega_{\mathrm{op}}\right)}} \\
& \times\left[1+2 \alpha\left(E-\hbar \omega_{\mathrm{op}}\right)\right] .
\end{aligned}
$$

Here, $\varepsilon_{\infty}$ and $\varepsilon_{0}$ are high-frequency and static dielectric constants, respectively. $\omega_{\mathrm{op}}$ is the optical phonon frequency and $\hbar \omega_{\mathrm{op}}$ is the optical phonon energy. For GaN, only the topmost non-dispersive LO phonon branch is considered, with its fixed $\hbar \omega_{\text {op }}$ of $91.2 \mathrm{meV}$. In addition, $m_{n}^{*}$ is the effective mass of electrons in the $n$-th valley, and $N_{q}$ is the occupation number for phonons with wave vector $\mathbf{q}$ given by $N_{q}=\left(e^{\hbar \omega / k_{B} T}-1\right)^{-1}$.

The temperature $T$ in the phonon occupation expression would be the lattice temperature when electrons and phonons are in thermal equilibrium. For a GaN device with high electric fields, hot electron effects happen, and such a thermal equilibrium is not reached. In this case, the temperature $T$ should be the temperature of optical phonons $\left(T_{O P}\right)$.

The electronic conduction band is approximated using the analytical nonparabolic form, given as

$$
\gamma_{n}(E)=E\left(1+\alpha_{n} E\right)=\frac{\hbar^{2} k^{2}}{2 m_{n}^{*}},
$$

where $\alpha_{n}$ is the nonparabolicity parameter for the $n$-th valley. 


\section{Acoustic phonon scattering}

Acoustic phonon scattering is calculated using the deformation potential. Given the acoustic deformation potential $D_{a}$, the scattering rate is

$$
\Gamma_{A C}(E)=\frac{k_{B} T D_{a}^{2}\left(m_{n}^{*}\right)^{3 / 2}}{\pi \hbar^{4} u_{l}^{2} \rho}\left(1+2 \alpha_{n} E\right) \sqrt{2 \gamma(E)},
$$

where $u_{l}$ is the longitudinal sound velocity and $\rho$ is the mass density.

Because of the low energy of acoustic phonons, acoustic phonon scattering is normally treated as elastic scattering, which means no change of electron energy before and after the scattering. ${ }^{54}$ However, the scattering still changes the velocity of an electron by randomly resetting the traveling direction of this electron. Adding the treatment of energy transfer in acoustic phonon scattering can be carried out in more detailed studies.

\section{Intervalley optical phonon scattering}

Intervalley scattering occurs when electrons from one valley are scattered into another valley. Normally, there is a large wave vector change for electrons to change their valley, and an optical phonon is needed to assist the scattering process. Intervalley optical phonon scattering is very important for high-energy electrons, such as the case for $\mathrm{GaN}$ under high electric fields. ${ }^{55}$ Using $D_{i j}$ as the deformation potential for an electron scattered from its initial $i$-th valley into the final $j$-th valley, the scattering rates are ${ }^{56}$

$$
\begin{aligned}
\Gamma_{I V}^{\mathrm{absorb}}(E)= & N_{q} \frac{D_{i j}^{2} Z_{i j}\left(m_{n}^{*}\right)^{\frac{3}{2}}}{\sqrt{2} \pi \hbar^{3} \rho \omega_{\mathrm{op}}}\left[1+2 \alpha_{n}\left(E+\hbar \omega_{\mathrm{op}}-\Delta E_{i j}\right)\right] \\
& \times \sqrt{\gamma\left(E+\hbar \omega_{\mathrm{op}}-\Delta E_{i j}\right)},
\end{aligned}
$$

and

$$
\begin{aligned}
\Gamma_{I V}^{\mathrm{emit}}(E)= & \left(N_{q}+1\right) \frac{D_{i j}^{2} Z_{i j}\left(m_{n}^{*}\right)^{\frac{3}{2}}}{\sqrt{2} \pi \hbar^{3} \rho \omega_{\mathrm{op}}}\left[1+2 \alpha_{n}\left(E-\hbar \omega_{\mathrm{op}}-\Delta E_{i j}\right)\right] \\
& \times \sqrt{\gamma\left(E-\hbar \omega_{\mathrm{op}}-\Delta E_{i j}\right)} .
\end{aligned}
$$

Here, $Z_{i j}$ is the number of equivalent intervalley routes and is equal to the product of the numbers of the equivalent valleys for the $i$-th and $j$-th valleys. $\Delta E_{i j}$ is the energy difference between the bottom of the $j$-th valley and the bottom of the $i$-th valley.

\section{APPENDIX B: BOUNDARY CONDITIONS FOR THE POISSON EQUATION SOLVER}

Polarization effects are strong for GaN and its ternary alloys, and they play a critical role in the formation of the extraordinarily high density 2DEG on AlGaN/GaN heterojunctions. The electric field applied to the 2DEG is important for electron MC simulations, and it can be calculated from the electric potential distribution around the 2DEG region obtained by solving the Poisson equation. Let $\Phi$ be the electric potential field, $\rho_{e}$ be the charge density, and $\varepsilon$ be the permittivity of the media. The Poisson equation is

$$
\nabla^{2} \Phi=-\frac{\rho_{e}}{\varepsilon} .
$$

Once the boundary condition and the charge distribution are given, the Poisson equation can be solved using standard tools and the electric field can be derived as $\mathcal{E}=-\nabla \Phi$.

Figure 7 shows the diagram of the 2D computational domain for the Poisson equation solver. Since 2DEG only resides at the $\mathrm{AlGaN} / \mathrm{GaN}$ interface, only these two layers are considered. $\mathrm{Al}_{0.3} \mathrm{Ga}_{0.7} \mathrm{~N}$ with a thickness of $30 \mathrm{~nm}$ is the top layer, and $2.5-\mu \mathrm{m}$-thick $\mathrm{GaN}$ is the bottom layer. The width of the simulation domain is $20 \mu \mathrm{m}$, which is much larger than the source-drain distance. The three thick line segments on top of the AlGaN layer indicate positions of the source, gate, and drain of the HEMT. The charge distribution on the surfaces and interface are input parameters for the Poisson equation solver. The fixed polarization charges are indicated in Fig. 7 with red plus and minus signs, while the mobile 2DEG is indicated with green minus signs inside circles. The net charge on the exposed upper surface of the $\mathrm{AlGaN}$ layer can be treated as zero, as this surface is normally passivated, ${ }^{57}$ or the polarization charge can be offset by the surface charge. ${ }^{58}$ The charge on the bottom surface of the $\mathrm{GaN}$ layer (i.e., the $\mathrm{GaN}-\mathrm{SiC}$ interface) can be ignored as it is far from the $\mathrm{AlGaN} / \mathrm{GaN}$ interface and its effect on the electric field in 2DEG is minimal. Based on these assumptions, the boundary condition for the Poisson equation is setting electric potential to be $\Phi_{\mathrm{S}}, \Phi_{\mathrm{G}}$, and $\Phi_{\mathrm{D}}$ for the segments of source, gate, and drain, respectively. For the rest part of the domain boundary, $\nabla \Phi \cdot \hat{n}=0$ is assigned, where the unit vector $\hat{n}$ indicates the normal direction of the boundary.

For wurtzite group-III nitride, the two most important polarizations are piezoelectric and spontaneous polarizations,

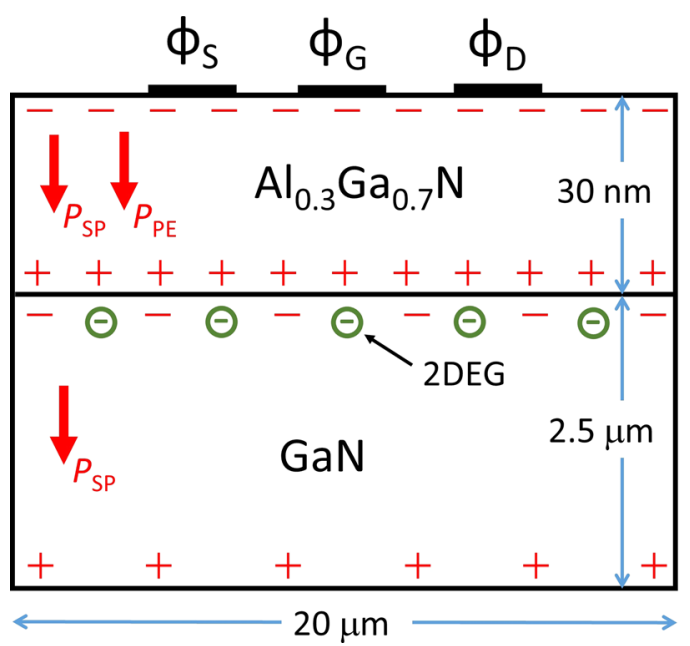

FIG. 7. (Not to scale.) Diagram of the computational domain for the Poisson equation solver. The AlGaN and GaN layers are considered, with 2DEG (indicated with green minus signs inside circles) at the GaN side of the interface. Polarization vectors are indicated with red thick arrows and polarization charges are indicated with red plus and minus signs. Thick line segments on top of the AlGaN layer indicate source, gate, and drain positions. The electric potential for these terminals is also indicated. 
denoted as $P_{\mathrm{PE}}$ and $P_{\mathrm{SP}}$, respectively. A detailed study with clear presentation was performed by Ambacher et al. ${ }^{59}$ on the polarization within $\mathrm{AlGaN}$ and GaN layers and the 2DEG formation at their interface. In this study, the material parameters used in the Poisson equation solver follow their work. ${ }^{59}$ Since normally a very thin AlGaN layer is grown on a thick GaN layer, the lattice relaxation happens on the AlGaN layer. In this situation, piezoelectric polarization (with tensile strain) is considered for the AlGaN layer only. On the other hand, spontaneous polarization exists for both $\mathrm{AlGaN}$ and $\mathrm{GaN}$ layers. This is also illustrated as red arrows in Fig. 7. Since polarization vectors are perpendicular to the AlGaN/GaN interface, the sheet polarization charge density can be calculated as the difference of the polarizations on the two sides of a surface. The material parameters from Ambacher et al. ${ }^{59}$ are $P_{\mathrm{SP}, \mathrm{GaN}}=2.9 \times 10^{-6} \mathrm{C} / \mathrm{cm}^{2}, \quad P_{\mathrm{SP}, \mathrm{AlGaN}}=4.5 \times 10^{-6} \mathrm{C} / \mathrm{cm}^{2}$, and $P_{\mathrm{PE}, \mathrm{AlGaN}}=1.1 \times 10^{-6} \mathrm{C} / \mathrm{cm}^{2}$ for $\mathrm{Al}_{0.3} \mathrm{Ga}_{0.7} \mathrm{~N}$. Thus, the sheet polarization charge density at the $\mathrm{AlGaN} / \mathrm{GaN}$ interface is $\sigma=\left(P_{\mathrm{SP}, \mathrm{AlGaN}}+P_{\mathrm{PE}, \mathrm{AlGaN}}\right)-P_{\mathrm{SP}, \mathrm{GaN}}=2.7 \times 10^{-6} \mathrm{C} / \mathrm{cm}^{2}$.

The sheet carrier charge density $\sigma_{e}$ for the $2 \mathrm{DEG}$ is obtained from electron MC simulations. In these simulations, the $\mathrm{AlGaN} / \mathrm{GaN}$ interface is divided into many unit cells as spatial bins to count the statistical electron properties. After the steady state is achieved, the electron number for each unit cell can be tallied and the sheet carrier charge density $\sigma_{e}$ can be calculated. In the Poisson solver, the overall sheet charge density at the AlGaN/GaN interface is the summation of $\sigma_{e}$ and the polarization charge density $\sigma$. Based on this overall sheet charge density and above-mentioned boundary conditions, the Poisson equation can be solved to derive the electric field for the 2DEG.

${ }^{1}$ Y.-F. Wu, M. Moore, A. Saxler, T. Wisleder, and P. Parikh, in 64th Device Research Conference (2006), p. 151.

${ }^{2}$ M. Yanagihara, Y. Uemoto, T. Ueda, T. Tanaka, and D. Ueda, Phys. Status Solidi A 206, 1221 (2009).

${ }^{3}$ Y.-R. Wu and J. Singh, J. Appl. Phys. 101, 113712 (2007).

${ }^{4}$ M. Rosker, C. Bozada, H. Dietrich, A. Hung, D. Via, S. Binari, E. Vivierios, E. Cohen, and J. Hodiak, in The International Conference on Compound Semiconductor Manufacturing Technology (Tampa, FL, 2009).

${ }^{5}$ R. S. Muller, T. I. Kamins, M. Chan, and P. K. Ko, Device Electronics for Integrated Circuits (Wiley, 1986).

${ }^{6}$ V. O. Turin and A. A. Balandin, J. Appl. Phys. 100, 054501 (2006).

${ }^{7}$ E. R. Heller and A. Crespo, Microelectron. Reliab. 48, 45 (2008).

${ }^{8}$ M. Garven and J. P. Calame, IEEE Trans. Compon. Packag. Technol. 32, 63 (2009).

${ }^{9}$ J. Cho, E. Bozorg-Grayeli, D. H. Altman, M. Asheghi, and K. E. Goodson, IEEE Electron Device Lett. 33, 378 (2012).

${ }^{10}$ H. Lee, D. D. Agonafer, Y. Won, F. Houshmand, C. Gorle, M. Asheghi, and K. E. Goodson, J. Electron. Packag. 138, 010907 (2016).

${ }^{11}$ P. G. Sverdrup, Y. S. Ju, and K. E. Goodson, J. Heat Transfer 123, 130 (2001).

${ }^{12}$ E. Pop, R. Dutton, and K. Goodson, in IEEE International Conference on Simulation of Semiconductor Processes and Devices (Boston, MA, USA, 2003), p. 121.

${ }^{13}$ E. Pop, S. Sinha, and K. E. Goodson, in ASME 2003 Heat Transfer Summer Conference (Las Vegas, NV, USA, 2003), p. 803.

${ }^{14}$ B. Benbakhti, A. Soltani, K. Kalna, M. Rousseau, and J. C. De Jaeger, IEEE Trans. Electron Devices 56, 2178 (2009).

${ }^{15}$ S. Vitanov, V. Palankovski, S. Maroldt, and R. Quay, Solid-State Electron. 54, 1105 (2010).

${ }^{16}$ A. Matulionis, J. Liberis, L. Ardaravičius, L. F. Eastman, J. R. Shealy, and A. Vertiatchikh, Semicond. Sci. Technol. 19, S421 (2004).

${ }^{17}$ B. K. Ridley, W. J. Schaff, and L. F. Eastman, J. Appl. Phys. 96, 1499 (2004).
${ }^{18}$ A. Fan, C. Tarau, R. Bonner, T. Palacios, and M. Kaviany, in ASME 2012 Summer Heat Transfer Conference (Rio Grande, Puerto Rico, USA, 2012), p. 685.

${ }^{19}$ A. Ashok, D. Vasileska, O. L. Hartin, and S. M. Goodnick, IEEE Trans. Electron Devices 57, 562 (2010).

${ }^{20}$ B. Padmanabhan, D. Vasileska, and S. M. Goodnick, J. Comput. Electron. 11, 129 (2012).

${ }^{21}$ T. Sadi, R. W. Kelsall, and N. J. Pilgrim, IEEE Trans. Electron Devices 53, 2892 (2006).

${ }^{22}$ T. Sadi, R. W. Kelsall, and N. J. Pilgrim, J. Comput. Electron. 6, 35 (2007).

${ }^{23}$ A. Matulionis, V. Aninkevičius, and M. Ramonas, Lith. J. Phys. 55, 335 (2015).

${ }^{24}$ A. Dyson, D. R. Naylor, and B. K. Ridley, IEEE Trans. Electron Devices 62, 3613 (2015).

${ }^{25}$ S. García, I. Íñiguez-de-la-Torre, J. Mateos, T. González, and S. Pérez, Semicond. Sci. Technol. 31, 065005 (2016).

${ }^{26}$ K. Maize, G. Pavlidis, E. Heller, L. Yates, D. Kendig, S. Graham, and A. Shakouri, in 2014 IEEE Compound Semiconductor Integrated Circuit Symposium (CSICS) (La Jolla, CA, USA, 2014), p. 1.

${ }^{27}$ O. Arenas, É. Al Alam, V. Aimez, A. Jaouad, H. Maher, R. Arès, and F. Boone, IEEE Electron Device Lett. 36, 111 (2015).

${ }^{28}$ H.-P. Chou, S. Cheng, C.-H. Cheng, and C.-W. Chuang, Mater. Sci. Semicond. Process. 41, 304 (2016).

${ }^{29}$ Q. Hao, G. Chen, and M.-S. Jeng, J. Appl. Phys. 106, 114321 (2009).

${ }^{30}$ E. O. Kane, J. Phys. Chem. Solids 1, 249 (1957).

${ }^{31}$ J. Kołodziejczak, Acta Phys. Pol. 20, 289 (1961).

${ }^{32}$ M. Lundstrom, Fundamentals of Carrier Transport, 2nd ed. (Cambridge University Press, New York, 2000).

${ }^{33}$ T. Hiroyoshi, Y. Naoki, T. Kenji, and H. Chihiro, Jpn. J. Appl. Phys., Part 127,563 (1988).

${ }^{34}$ M. Ramonas, A. Matulionis, and L. Rota, Semicond. Sci. Technol. 18, 118 (2003).

${ }^{35}$ T. Li, R. P. Joshi, and C. Fazi, J. Appl. Phys. 88, 829 (2000).

${ }^{36}$ W. Fawcett, A. D. Boardman, and S. Swain, J. Phys. Chem. Solids 31, 1963 (1970).

${ }^{37}$ S. B. Lisesivdin, A. Yildiz, N. Balkan, M. Kasap, S. Ozcelik, and E. Ozbay, J. Appl. Phys. 108, 013712 (2010).

${ }^{38}$ C. Bulutay, B. K. Ridley, and N. A. Zakhleniuk, Phys. Rev. B 62, 15754 (2000).

${ }^{39}$ J. Zhang, Y. Hao, J. Zhang, and J. Ni, Sci. China, Ser. F-Inf. Sci. 51, 780 (2008).

${ }^{40}$ X. Gang, E. Xu, N. Hashemi, Z. Bo, F. Y. Fu, and W. T. Ng, Chin. Phys. B 21, 086105 (2012).

${ }^{41} \mathrm{Q}$. Hao and G. Chen, in Applications of Monte Carlo Method in Science and Engineering, edited by S. Mordechai (InTech, 2011), p. 707.

${ }^{42}$ Q. Hao, J. Appl. Phys. 111, 014309 (2012).

${ }^{43}$ J.-P. M. Péraud and N. G. Hadjiconstantinou, Phys. Rev. B 84, 205331 (2011).

${ }^{44}$ J.-P. M. Péraud and N. G. Hadjiconstantinou, Appl. Phys. Lett. 101, 153114 (2012).

${ }^{45}$ C. Dames and G. Chen, in Thermoelectrics Handbook: Macro to Nano, edited by D. M. Rowe (CRC Press, Boca Raton, 2006), p. 42.01.

${ }^{46}$ B. A. Danilchenko, I. A. Obukhov, T. Paszkiewicz, S. Wolski, and A. Jeżowski, Solid State Commun. 144, 114 (2007).

${ }^{47}$ J. P. Freedman, J. H. Leach, E. A. Preble, Z. Sitar, R. F. Davis, and J. A. Malen, Sci. Rep. 3, 2963 (2013).

${ }^{48}$ J. Lai and A. Majumdar, J. Appl. Phys. 79, 7353 (1996).

${ }^{49}$ K. Raleva, D. Vasileska, S. M. Goodnick, and M. Nedjalkov, IEEE Trans. Electron Devices 55, 1306 (2008).

${ }^{50}$ J. Schleeh, J. Mateos, I. Iniguez-de-la-Torre, N. Wadefalk, P. A. Nilsson, J. Grahn, and A. J. Minnich, Nat. Mater. 14, 187 (2015).

${ }^{51}$ H. Fröhlich, Phys. Rev. 79, 845 (1950).

${ }^{52}$ L. Hsu and W. Walukiewicz, Phys. Rev. B 56, 1520 (1997).

${ }^{53}$ T.-H. Yu and K. F. Brennan, J. Appl. Phys. 91, 3730 (2002).

${ }^{54}$ C. Jacoboni and P. Lugli, The Monte Carlo Method for Semiconductor Device Simulation (Springer-Verlag, New York, 1989).

${ }^{55}$ B. Gelmont, K. Kim, and M. Shur, J. Appl. Phys. 74, 1818 (1993).

${ }^{56}$ B. K. Ridley, Quantum Processes in Semiconductors, 2nd ed. (Oxford University Press, Oxford, 1988).

${ }^{57}$ S. Russo and A. Di Carlo, IEEE Trans. Electron Devices 54, 1071 (2007).

${ }^{58}$ J. Si, J. Wei, W. Chen, and B. Zhang, IEEE Trans. Electron Devices 60, 3223 (2013).

${ }^{59}$ O. Ambacher, J. Smart, J. R. Shealy, N. G. Weimann, K. Chu, M. Murphy, W. J. Schaff, L. F. Eastman, R. Dimitrov, and L. Wittmer, J. Appl. Phys. 85, 3222 (1999). 